Network Working Group

Request for Comments: 2049

Obsoletes: 1521, 1522, 1590

Category: Standards Track
N. Freed

Innosoft

N. Borenstein

First Virtual

November 1996

\title{
Multipurpose Internet Mail Extensions \\ (MIME) Part Five: \\ Conformance Criteria and Examples
}

Status of this Memo

This document specifies an Internet standards track protocol for the Internet community, and requests discussion and suggestions for improvements. Please refer to the current edition of the "Internet Official Protocol Standards" (STD 1) for the standardization state and status of this protocol. Distribution of this memo is unlimited.

\section{Abstract}

STD 11, RFC 822, defines a message representation protocol specifying considerable detail about US-ASCII message headers, and leaves the message content, or message body, as flat US-ASCII text. This set of documents, collectively called the Multipurpose Internet Mail

Extensions, or MIME, redefines the format of messages to allow for textual message bodies in character sets other than US-ASCII, an extensible set of different formats for non-textual message bodies,

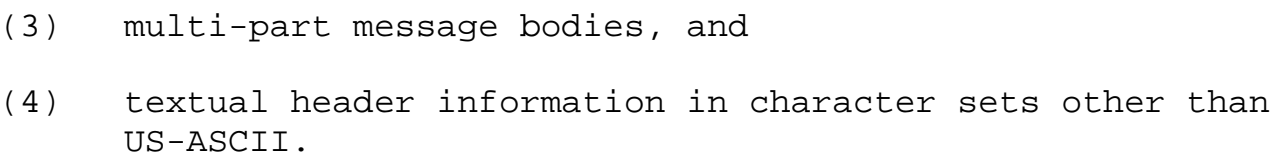

These documents are based on earlier work documented in RFC 934, STD 11, and RFC 1049, but extends and revises them. Because RFC 822 said so little about message bodies, these documents are largely orthogonal to (rather than a revision of) RFC 822 .

The initial document in this set, RFC 2045, specifies the various headers used to describe the structure of MIME messages. The second document defines the general structure of the MIME media typing system and defines an initial set of media types. The third document, RFC 2047, describes extensions to RFC 822 to allow non-US- 
ASCII text data in Internet mail header fields. The fourth document, RFC 2048, specifies various IANA registration procedures for MIMErelated facilities. This fifth and final document describes MIME conformance criteria as well as providing some illustrative examples of MIME message formats, acknowledgements, and the bibliography.

These documents are revisions of RFCs 1521, 1522, and 1590, which themselves were revisions of RFCs 1341 and 1342. Appendix B of this document describes differences and changes from previous versions.

Table of Contents

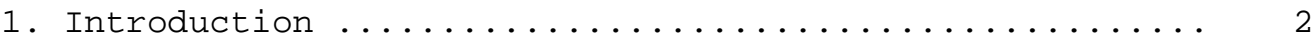

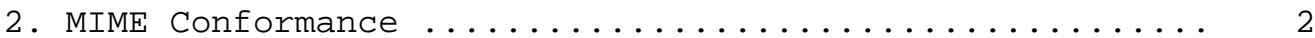

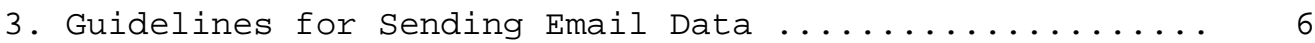

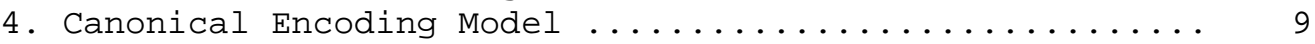

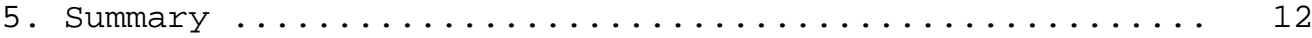

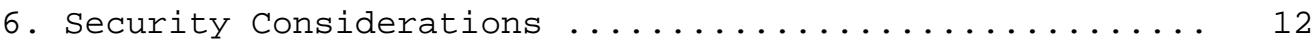

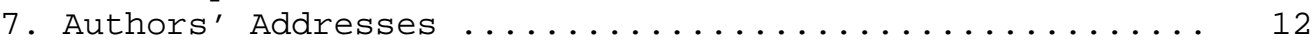

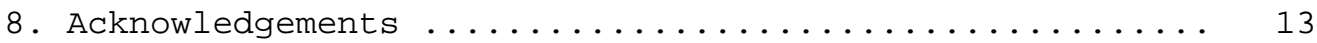

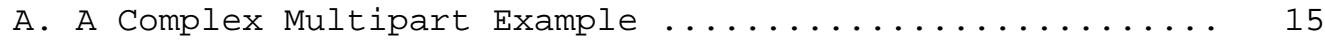

B. Changes from RFC 1521, 1522, and $1590 \ldots \ldots \ldots \ldots \ldots$

C. References ............................ 20

1. Introduction

The first and second documents in this set define MIME header fields and the initial set of MIME media types. The third document describes extensions to RFC822 formats to allow for character sets other than US-ASCII. This document describes what portions of MIME must be supported by a conformant MIME implementation. It also describes various pitfalls of contemporary messaging systems as well as the canonical encoding model MIME is based on.

\section{MIME Conformance}

The mechanisms described in these documents are open-ended. It is definitely not expected that all implementations will support all available media types, nor that they will all share the same extensions. In order to promote interoperability, however, it is useful to define the concept of "MIME-conformance" to define a certain level of implementation that allows the useful interworking of messages with content that differs from US-ASCII text. In this section, we specify the requirements for such conformance. 
A mail user agent that is MIME-conformant MUST:

(1) Always generate a "MIME-Version: 1.0" header field in any message it creates.

(2) Recognize the Content-Transfer-Encoding header field and decode all received data encoded by either quotedprintable or base64 implementations. The identity transformations 7bit, 8bit, and binary must also be recognized.

Any non-7bit data that is sent without encoding must be properly labelled with a content-transfer-encoding of 8bit or binary, as appropriate. If the underlying transport does not support 8bit or binary (as SMTP [RFC-821] does not), the sender is required to both encode and label data using an appropriate contentTransfer-Encoding such as quoted-printable or base64.

(3) Must treat any unrecognized Content-Transfer-Encoding as if it had a Content-Type of "application/octetstream", regardless of whether or not the actual Content-Type is recognized.

(4) Recognize and interpret the Content-Type header field, and avoid showing users raw data with a Content-Type field other than text. Implementations must be able to send at least text/plain messages, with the character set specified with the charset parameter if it is not US-ASCII.

(5) Ignore any content type parameters whose names they do not recognize.

(6) Explicitly handle the following media type values, to at least the following extents:

Text:

-- Recognize and display "text" mail with the character set "US-ASCII."

-- Recognize other character sets at least to the extent of being able to inform the user about what character set the message uses. 


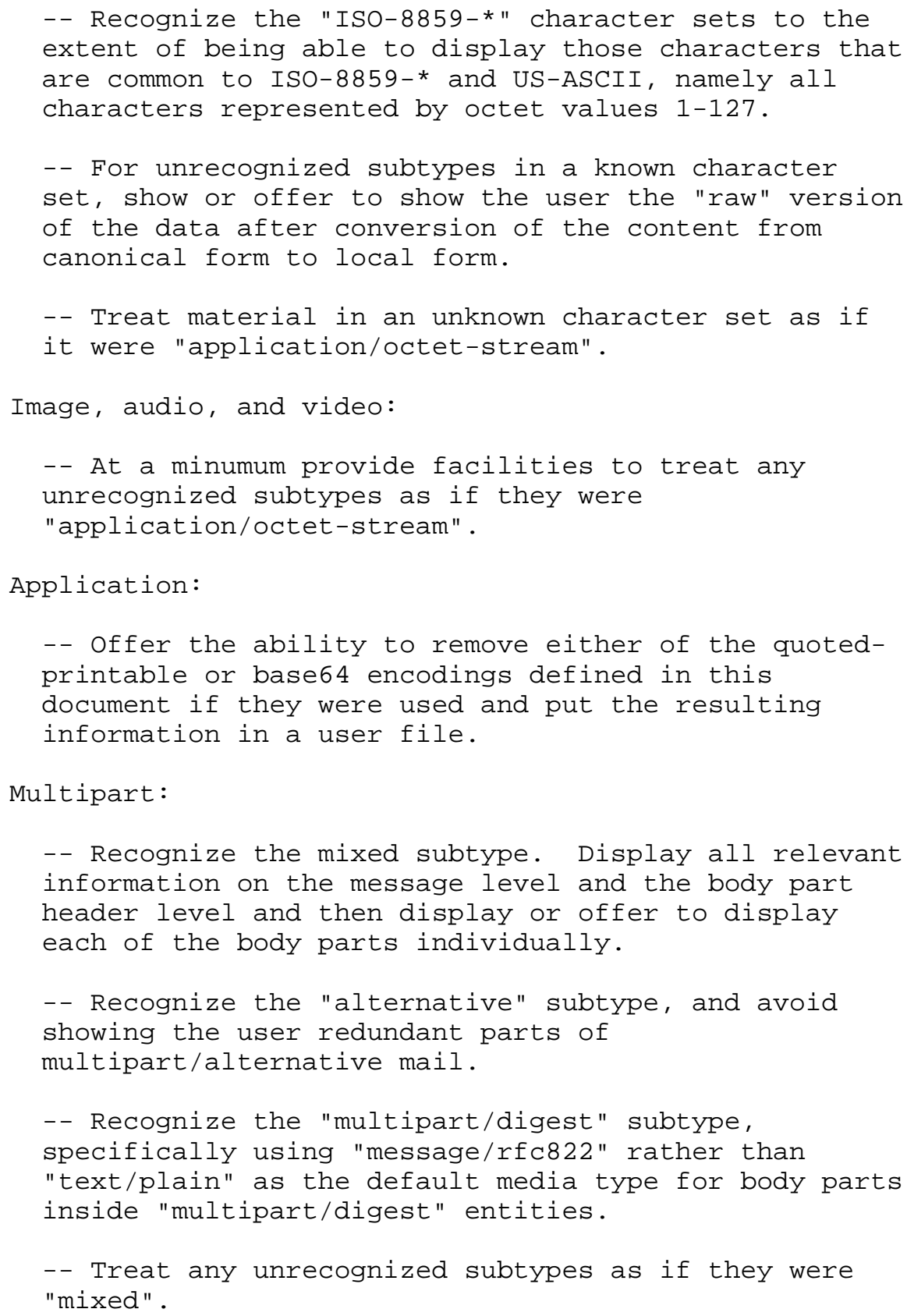


Message:

-- Recognize and display at least the RFC822 message encapsulation (message/rfc822) in such a way as to preserve any recursive structure, that is, displaying or offering to display the encapsulated data in accordance with its media type.

-- Treat any unrecognized subtypes as if they were "application/octet-stream".

(7) Upon encountering any unrecognized Content-Type field, an implementation must treat it as if it had a media type of "application/octet-stream" with no parameter sub-arguments. How such data are handled is up to an implementation, but likely options for handling such unrecognized data include offering the user to write it into a file (decoded from its mail transport format) or offering the user to name a program to which the decoded data should be passed as input. non-standard support for non-MIME messages employing character sets other than US-ASCII, to do so on received messages only. Conforming user agents must not send non-MIME messages containing anything other than US-ASCII text.

In particular, the use of non-US-ASCII text in mail messages without a MIME-Version field is strongly discouraged as it impedes interoperability when sending messages between regions with different localization conventions. Conforming user agents MUST include proper MIME labelling when sending anything other than plain text in the US-ASCII character set.

In addition, non-MIME user agents should be upgraded if at all possible to include appropriate MIME header information in the messages they send even if nothing else in MIME is supported. This upgrade will have little, if any, effect on non-MIME recipients and will aid MIME in correctly displaying such messages. It also provides a smooth transition path to eventual adoption of other MIME capabilities.

(9) Conforming user agents must ensure that any string of non-white-space printable US-ASCII characters within a "*text" or "*ctext" that begins with "=?" and ends with 
"?=" be a valid encoded-word. ("begins" means: At the start of the field-body or immediately following linear-white-space; "ends" means: At the end of the field-body or immediately preceding linear-whitespace.) In addition, any "word" within a "phrase" that begins with "=?" and ends with "?=" must be a valid encoded-word.

(10) Conforming user agents must be able to distinguish encoded-words from "text", "ctext", or "word"s, according to the rules in section 4, anytime they appear in appropriate places in message headers. It must support both the "B" and "Q" encodings for any character set which it supports. The program must be able to display the unencoded text if the character set is "US-ASCII". For the ISO-8859-* character sets, the mail reading program must at least be able to display the characters which are also in the US-ASCII set.

A user agent that meets the above conditions is said to be MIMEconformant. The meaning of this phrase is that it is assumed to be "safe" to send virtually any kind of properly-marked data to users of such mail systems, because such systems will at least be able to treat the data as undifferentiated binary, and will not simply splash it onto the screen of unsuspecting users.

There is another sense in which it is always "safe" to send data in a format that is MIME-conformant, which is that such data will not break or be broken by any known systems that are conformant with RFC 821 and RFC 822. User agents that are MIME-conformant have the additional guarantee that the user will not be shown data that were never intended to be viewed as text.

\section{Guidelines for Sending Email Data}

Internet email is not a perfect, homogeneous system. Mail may become corrupted at several stages in its travel to a final destination. Specifically, email sent throughout the Internet may travel across many networking technologies. Many networking and mail technologies do not support the full functionality possible in the SMTP transport environment. Mail traversing these systems is likely to be modified in order that it can be transported.

There exist many widely-deployed non-conformant MTAs in the Internet. These MTAs, speaking the SMTP protocol, alter messages on the fly to take advantage of the internal data structure of the hosts they are implemented on, or are just plain broken. 
The following guidelines may be useful to anyone devising a data format (media type) that is supposed to survive the widest range of networking technologies and known broken MTAs unscathed. Note that anything encoded in the base64 encoding will satisfy these rules, but that some well-known mechanisms, notably the UNIX uuencode facility, will not. Note also that anything encoded in the Quoted-Printable encoding will survive most gateways intact, but possibly not some gateways to systems that use the EBCDIC character set.

(1) Under some circumstances the encoding used for data may change as part of normal gateway or user agent operation. In particular, conversion from base64 to quoted-printable and vice versa may be necessary. This may result in the confusion of CRLF sequences with line breaks in text bodies. As such, the persistence of CRLF as something other than a line break must not be relied on.

(2) Many systems may elect to represent and store text data using local newline conventions. Local newline conventions may not match the RFC822 CRLF convention -systems are known that use plain CR, plain LF, CRLF, or counted records. The result is that isolated CR and LF characters are not well tolerated in general; they may be lost or converted to delimiters on some systems, and hence must not be relied on.

The transmission of NULs (US-ASCII value 0) is problematic in Internet mail. (This is largely the result of NULs being used as a termination character by many of the standard runtime library routines in the $\mathrm{C}$ programming language.) The practice of using NULs as termination characters is so entrenched now that messages should not rely on them being preserved.

(4) TAB (HT) characters may be misinterpreted or may be automatically converted to variable numbers of spaces. This is unavoidable in some environments, notably those not based on the US-ASCII character set. Such conversion is STRONGLY DISCOURAGED, but it may occur, and mail formats must not rely on the persistence of $\mathrm{TAB}(\mathrm{HT})$ characters.

(5) Lines longer than 76 characters may be wrapped or truncated in some environments. Line wrapping or line truncation imposed by mail transports is STRONGLY DISCOURAGED, but unavoidable in some cases. Applications which require long lines must somehow 
differentiate between soft and hard line breaks. (A simple way to do this is to use the quoted-printable encoding.)

Trailing "white space" characters (SPACE, TAB (HT)) on a line may be discarded by some transport agents, while other transport agents may pad lines with these characters so that all lines in a mail file are of equal length. The persistence of trailing white space, therefore, must not be relied on.

(7) Many mail domains use variations on the US-ASCII character set, or use character sets such as EBCDIC which contain most but not all of the US-ASCII characters. The correct translation of characters not in the "invariant" set cannot be depended on across character converting gateways. For example, this situation is a problem when sending uuencoded information across BITNET, an EBCDIC system. Similar problems can occur without crossing a gateway, since many Internet hosts use character sets other than USASCII internally. The definition of Printable strings in X.400 adds further restrictions in certain special cases. In particular, the only characters that are known to be consistent across all gateways are the 73 characters that correspond to the upper and lower case letters $A-z$ and $a-z$, the 10 digits 0-9, and the following eleven special characters:

$$
\begin{aligned}
& \text { " " (US-ASCII decimal value 39) } \\
& \text { "(" (US-ASCII decimal value 40) } \\
& \text { ") " (US-ASCII decimal value 41) } \\
& \text { "+" (US-ASCII decimal value 43) } \\
& \text { "," (US-ASCII decimal value 44) } \\
& \text { "-" (US-ASCII decimal value 45) } \\
& \text { "." (US-ASCII decimal value 46) } \\
& \text { "/" (US-ASCII decimal value 47) } \\
& \text { ":" (US-ASCII decimal value 58) } \\
& "=" \text { (US-ASCII decimal value 61) } \\
& \text { "?" (US-ASCII decimal value 63) }
\end{aligned}
$$

A maximally portable mail representation will confine itself to relatively short lines of text in which the only meaningful characters are taken from this set of 73 characters. The base64 encoding follows this rule.

(8) Some mail transport agents will corrupt data that includes certain literal strings. In particular, a 
period (".") alone on a line is known to be corrupted by some (incorrect) SMTP implementations, and a line that starts with the five characters "From " (the fifth character is a SPACE) are commonly corrupted as well. A careful composition agent can prevent these corruptions by encoding the data (e.g., in the quotedprintable encoding using "=46rom " in place of "From " at the start of a line, and "=2E" in place of "." alone on a line).

Please note that the above list is NOT a list of recommended practices for MTAs. RFC 821 MTAs are prohibited from altering the character of white space or wrapping long lines. These BAD and invalid practices are known to occur on established networks, and implementations should be robust in dealing with the bad effects they can cause.

4. Canonical Encoding Model

There was some confusion, in earlier versions of these documents, regarding the model for when email data was to be converted to canonical form and encoded, and in particular how this process would affect the treatment of CRLFs, given that the representation of newlines varies greatly from system to system. For this reason, a canonical model for encoding is presented below.

The process of composing a MIME entity can be modeled as being done in a number of steps. Note that these steps are roughly similar to those steps used in PEM [RFC-1421] and are performed for each "innermost level" body:

(1) Creation of local form.

The body to be transmitted is created in the system's native format. The native character set is used and, where appropriate, local end of line conventions are used as well. The body may be a UNIX-style text file, or a sun raster image, or a VMS indexed file, or audio data in a system-dependent format stored only in memory, or anything else that corresponds to the local model for the representation of some form of information. Fundamentally, the data is created in the "native" form that corresponds to the type specified by the media type. 
Conversion to canonical form.

The entire body, including "out-of-band" information such as record lengths and possibly file attribute information, is converted to a universal canonical form. The specific media type of the body as well as its associated attributes dictate the nature of the canonical form that is used. Conversion to the proper canonical form may involve character set conversion, transformation of audio data, compression, or various other operations specific to the various media types. If character set conversion is involved, however, care must be taken to understand the semantics of the media type, which may have strong implications for any character set conversion, e.g. with regard to syntactically meaningful characters in a text subtype other than "plain".

For example, in the case of text/plain data, the text must be converted to a supported character set and lines must be delimited with CRLF delimiters in accordance with $\mathrm{RFC}$ 822. Note that the restriction on line lengths implied by RFC 822 is eliminated if the next step employs either quoted-printable or base64 encoding.

Apply transfer encoding.

A Content-Transfer-Encoding appropriate for this body is applied. Note that there is no fixed relationship between the media type and the transfer encoding. In particular, it may be appropriate to base the choice of base64 or quoted-printable on character frequency counts which are specific to a given instance of a body .

(4) Insertion into entity.

The encoded body is inserted into a MIME entity with appropriate headers. The entity is then inserted into the body of a higher-level entity (message or multipart) as needed.

Conversion from entity form to local form is accomplished by reversing these steps. Note that reversal of these steps may produce differing results since there is no guarantee that the original and final local forms are the same. 
It is vital to note that these steps are only a model; they are specifically NOT a blueprint for how an actual system would be built. In particular, the model fails to account for two common designs:

In many cases the conversion to a canonical form prior to encoding will be subsumed into the encoder itself, which understands local formats directly. For example, the local newline convention for text bodies might be carried through to the encoder itself along with knowledge of what that format is.

(2) The output of the encoders may have to pass through one or more additional steps prior to being transmitted as a message. As such, the output of the encoder may not be conformant with the formats specified by RFC 822 . In particular, once again it may be appropriate for the converter's output to be expressed using local newline conventions rather than using the standard RFC $822 \mathrm{CRLF}$ delimiters.

Other implementation variations are conceivable as well. The vital aspect of this discussion is that, in spite of any optimizations, collapsings of required steps, or insertion of additional processing, the resulting messages must be consistent with those produced by the model described here. For example, a message with the following header fields:

Content-type: text/foo; charset=bar

Content-Transfer-Encoding: base 64

must be first represented in the text/foo form, then (if necessary) represented in the "bar" character set, and finally transformed via the base64 algorithm into a mail-safe form.

NOTE: Some confusion has been caused by systems that represent messages in a format which uses local newline conventions which differ from the RFC822 CRLF convention. It is important to note that these formats are not canonical RFC822/MIME. These formats are instead *encodings* of RFC822, where CRLF sequences in the canonical representation of the message are encoded as the local newline convention. Note that formats which encode CRLF sequences as, for example, LF are not capable of representing MIME messages containing binary data which contains LF octets not part of CRLF line separation sequences. 
5. Summary

This document defines what is meant by MIME Conformance. It also details various problems known to exist in the Internet email system and how to use MIME to overcome them. Finally, it describes MIME's canonical encoding model.

6. Security Considerations

Security issues are discussed in the second document in this set, RFC 2046 .

\section{Authors' Addresses}

For more information, the authors of this document are best contacted via Internet mail:

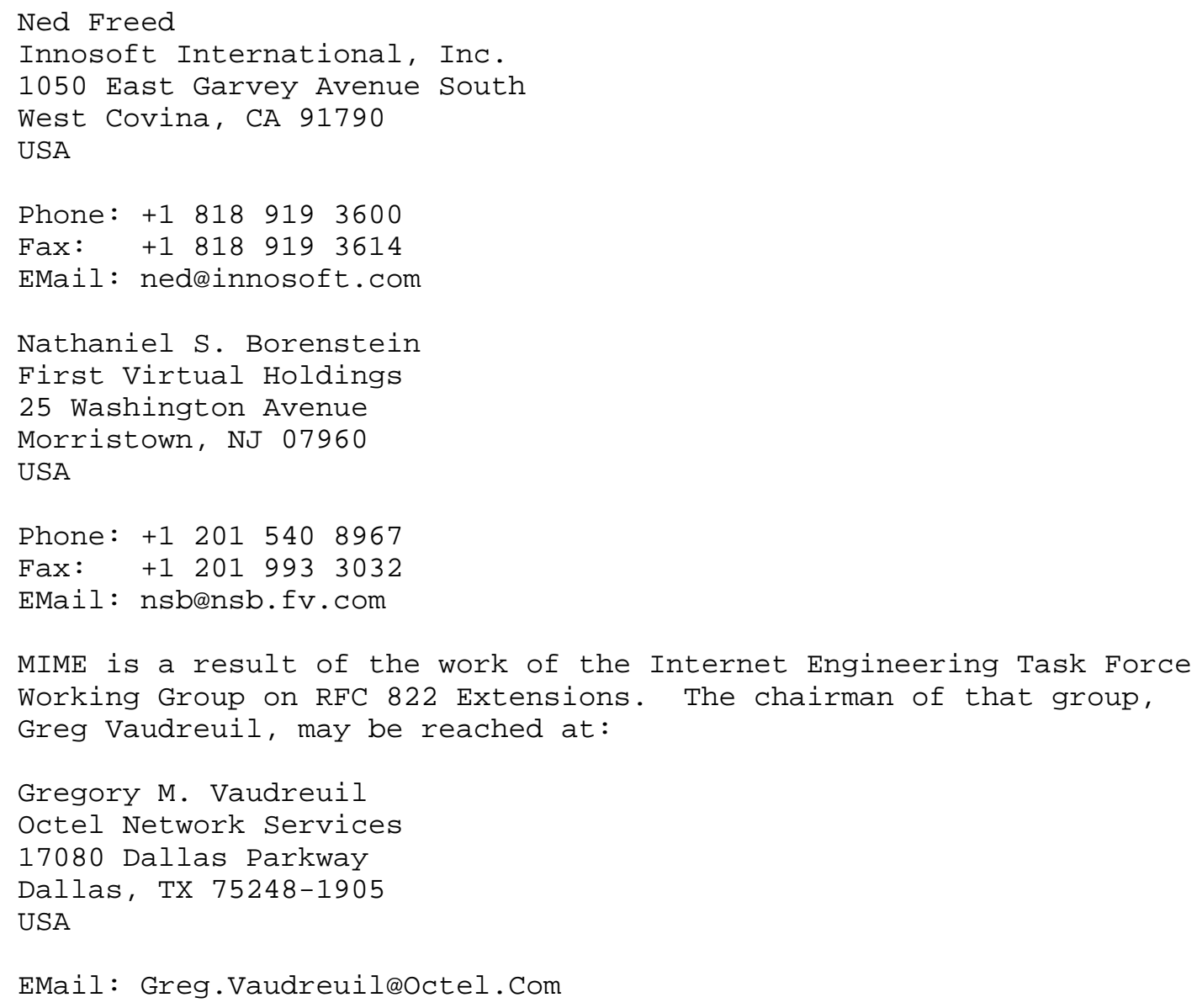




\section{Acknowledgements}

This document is the result of the collective effort of a large number of people, at several IETF meetings, on the IETF-SMTP and IETF-822 mailing lists, and elsewhere. Although any enumeration seems doomed to suffer from egregious omissions, the following are among the many contributors to this effort:

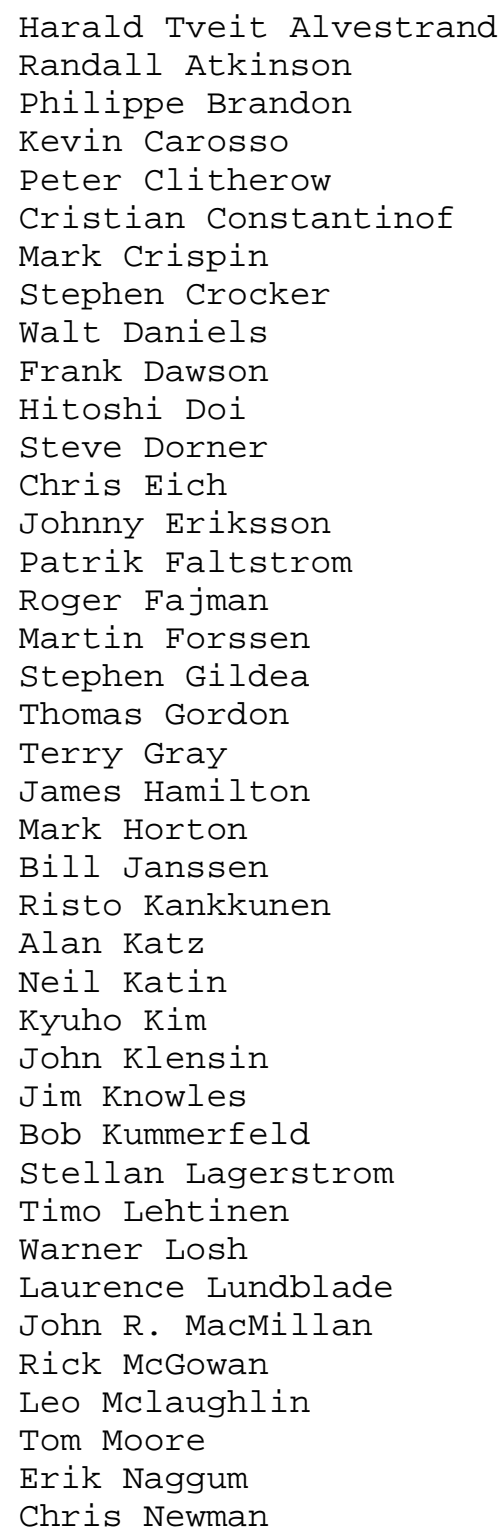

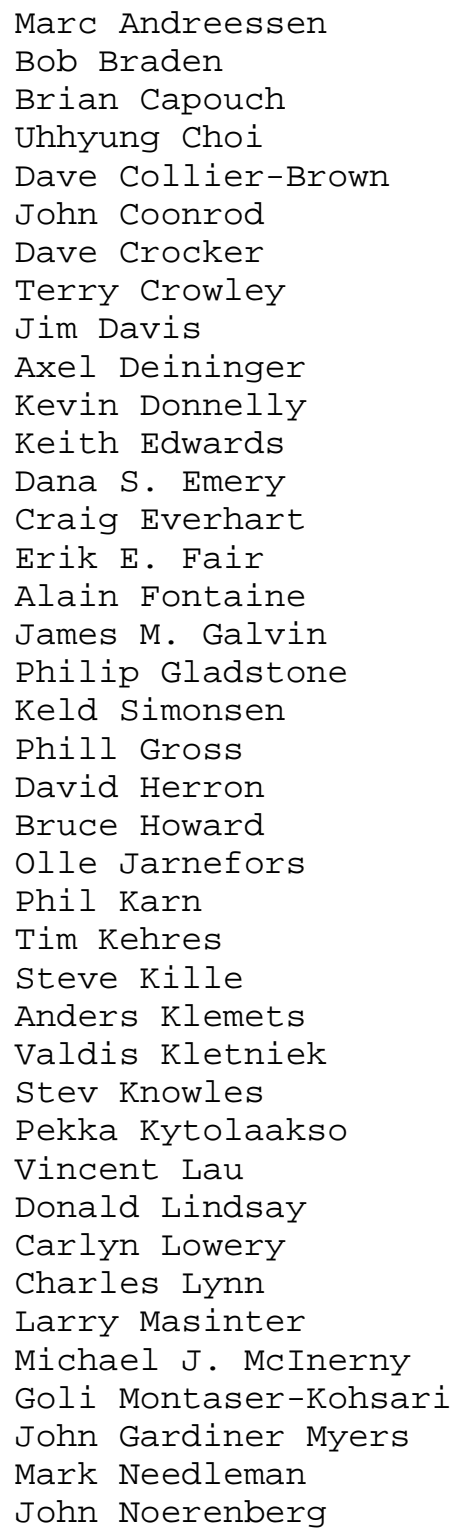




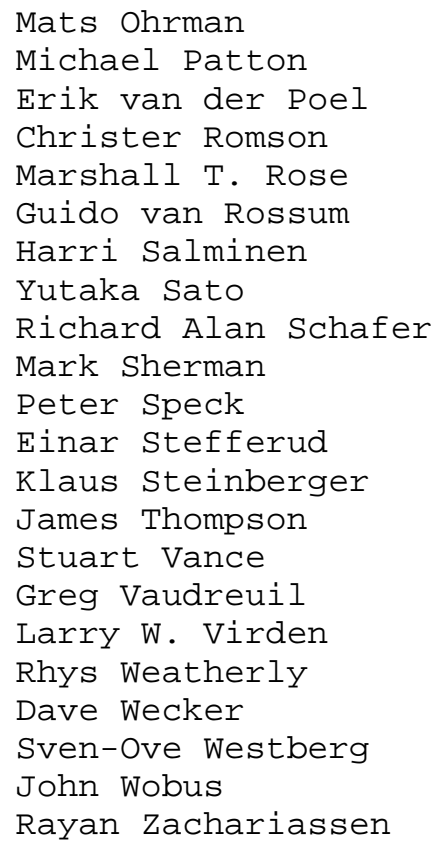

The authors apologize for any omissions from this list, which are certainly unintentional. 


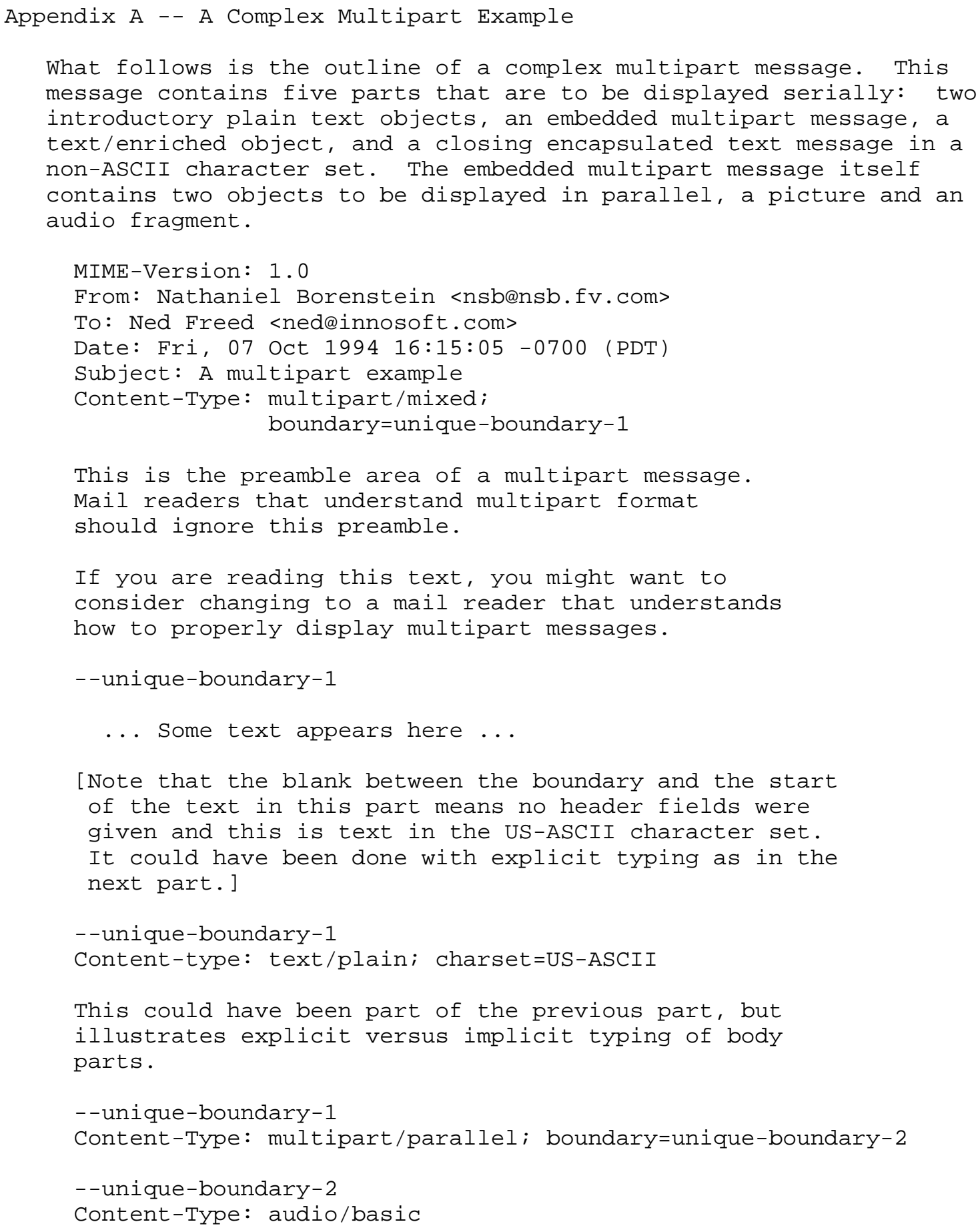




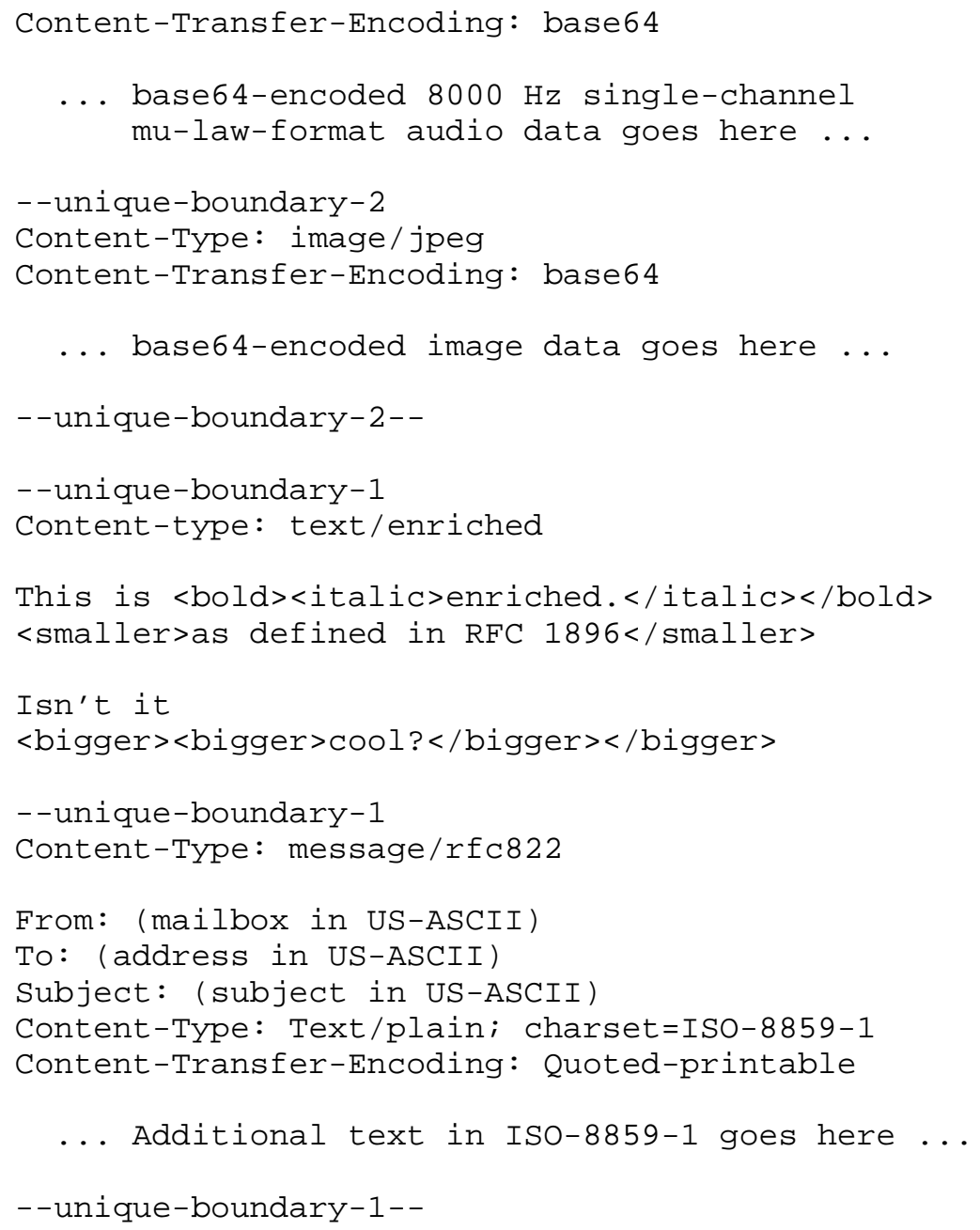

(1) This document has been completely reformatted and split into multiple documents. This was done to improve the quality of the plain text version of this document, which is required to be the reference copy. 
(2) BNF describing the overall structure of MIME object headers has been added. This is a documentation change only -- the underlying syntax has not changed in any way.

(3) The specific BNF for the seven media types in MIME has been removed. This BNF was incorrect, incomplete, amd inconsistent with the type-indendependent BNF. And since the type-independent BNF already fully specifies the syntax of the various MIME headers, the typespecific BNF was, in the final analysis, completely unnecessary and caused more problems than it solved.

(4) The more specific "US-ASCII" character set name has replaced the use of the informal term ASCII in many parts of these documents.

(5) The informal concept of a primary subtype has been removed.

(6) The term "object" was being used inconsistently. The definition of this term has been clarified, along with the related terms "body", "body part", and "entity", and usage has been corrected where appropriate.

(7) The BNF for the multipart media type has been rearranged to make it clear that the CRLF preceeding the boundary marker is actually part of the marker itself rather than the preceeding body part.

(8) The prose and BNF describing the multipart media type have been changed to make it clear that the body parts within a multipart object MUST NOT contain any lines beginning with the boundary parameter string.

(9) In the rules on reassembling "message/partial" MIME entities, "Subject" is added to the list of headers to take from the inner message, and the example is modified to clarify this point.

(10) "Message/partial" fragmenters are restricted to splitting MIME objects only at line boundaries.

(11) In the discussion of the application/postscript type, an additional paragraph has been added warning about possible interoperability problems caused by embedding of binary data inside a Postscript MIME entity. 
(12) Added a clarifying note to the basic syntax rules for the Content-Type header field to make it clear that the following two forms:

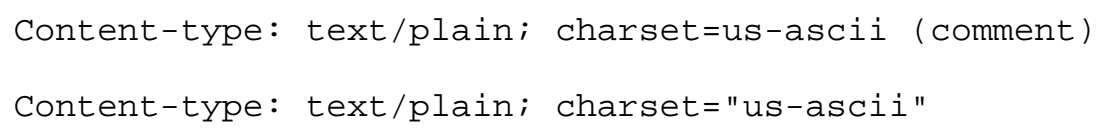

(13) The following sentence has been removed from the discussion of the MIME-Version header: "However, conformant software is encouraged to check the version number and at least warn the user if an unrecognized MIME-version is encountered."

(14) A typo was fixed that said "application/external-body" instead of "message/external-body".

(15) The definition of a character set has been reorganized to make the requirements clearer.

(16) The definition of the "image/gif" media type has been moved to a separate document. This change was made because of potential conflicts with IETF rules governing the standardization of patented technology.

(17) The definitions of "7bit" and "8bit" have been tightened so that use of bare CR, LF can only be used as end-of-line sequences. The document also no longer requires that NUL characters be preserved, which brings MIME into alignment with real-world implementations.

(18) The definition of canonical text in MIME has been tightened so that line breaks must be represented by a CRLF sequence. $\quad \mathrm{CR}$ and LF characters are not allowed outside of this usage. The definition of quotedprintable encoding has been altered accordingly.

(19) The definition of the quoted-printable encoding now includes a number of suggestions for how quotedprintable encoders might best handle improperly encoded material.

Prose was added to clarify the use of the "7bit", "8bit", and "binary" transfer-encodings on multipart or message entities encapsulating "8bit" or "binary" data. 
(21) In the section on MIME Conformance, "multipart/digest" support was added to the list of requirements for minimal MIME conformance. Also, the requirement for "message/rfc822" support were strengthened to clarify the importance of recognizing recursive structure.

(22) The various restrictions on subtypes of "message" are now specified entirely on a subtype by subtype basis.

(23) The definition of "message/rfc822" was changed to indicate that at least one of the "From", "Subject", or "Date" headers must be present.

(24) The required handling of unrecognized subtypes as "application/octet-stream" has been made more explicit in both the type definitions sections and the conformance guidelines.

(25) Examples using text/richtext were changed to text/enriched.

(26) The BNF definition of subtype has been changed to make it clear that either an IANA registered subtype or a nonstandard "X-" subtype must be used in a Content-Type header field.

(27) MIME media types that are simply registered for use and those that are standardized by the IETF are now distinguished in the MIME BNF.

(28) All of the various MIME registration procedures have been extensively revised. IANA registration procedures for character sets have been moved to a separate document that is no included in this set of documents.

(29) The use of escape and shift mechanisms in the US-ASCII and ISO-8859-X character sets these documents define have been clarified: Such mechanisms should never be used in conjunction with these character sets and their effect if they are used is undefined.

(30) The definition of the AFS access-type for message/external-body has been removed.

(31) The handling of the combination of multipart/alternative and message/external-body is now specifically addressed. 
(32) Security issues specific to message/external-body are now discussed in some detail.

Appendix C -- References

[ATK]

Borenstein, Nathaniel S., Multimedia Applications

Development with the Andrew Toolkit, Prentice-Hall, 1990.

[ ISO-2022]

International Standard -- Information Processing -Character Code Structure and Extension Techniques, ISO/IEC 2022:1994, 4th ed.

[ ISO-8859]

International Standard -- Information Processing -- 8-bit Single-Byte Coded Graphic Character Sets

- Part 1: Latin Alphabet No. 1, ISO 8859-1:1987, 1st ed.

- Part 2: Latin Alphabet No. 2, ISO 8859-2:1987, 1st ed.

- Part 3: Latin Alphabet No. 3, ISO 8859-3:1988, 1st ed.

- Part 4: Latin Alphabet No. 4, ISO 8859-4:1988, 1st ed.

- Part 5: Latin/Cyrillic Alphabet, ISO 8859-5:1988, 1st ed.

- Part 6: Latin/Arabic Alphabet, ISO 8859-6:1987, 1st ed.

- Part 7: Latin/Greek Alphabet, ISO 8859-7:1987, 1st ed.

- Part 8: Latin/Hebrew Alphabet, ISO 8859-8:1988, 1st ed.

- Part 9: Latin Alphabet No. 5, ISO/IEC 8859-9:1989, 1st ed.

International Standard -- Information Technology -- 8-bit Single-Byte Coded Graphic Character Sets

- Part 10: Latin Alphabet No. 6, ISO/IEC 8859-10:1992, 1st ed.

[ ISO-646]

International Standard -- Information Technology -- ISO

7-bit Coded Character set for Information Interchange, ISO 646:1991, 3rd ed..

[ JPEG ]

JPEG Draft Standard ISO 10918-1 CD.

[MPEG]

Video Coding Draft Standard ISO 11172 CD, ISO

IEC/JTC1/SC2/WG11 (Motion Picture Experts Group), May, 1991 . 


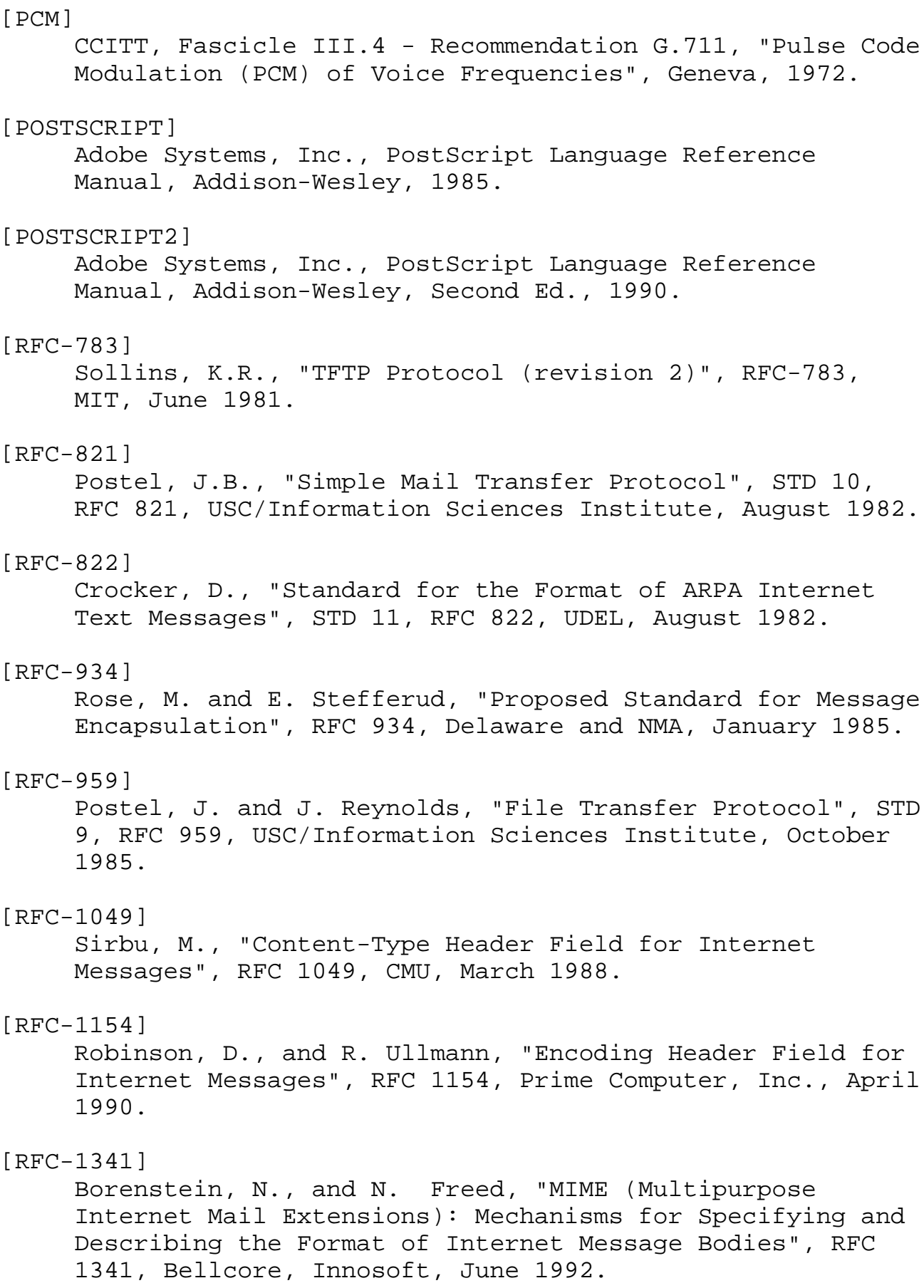




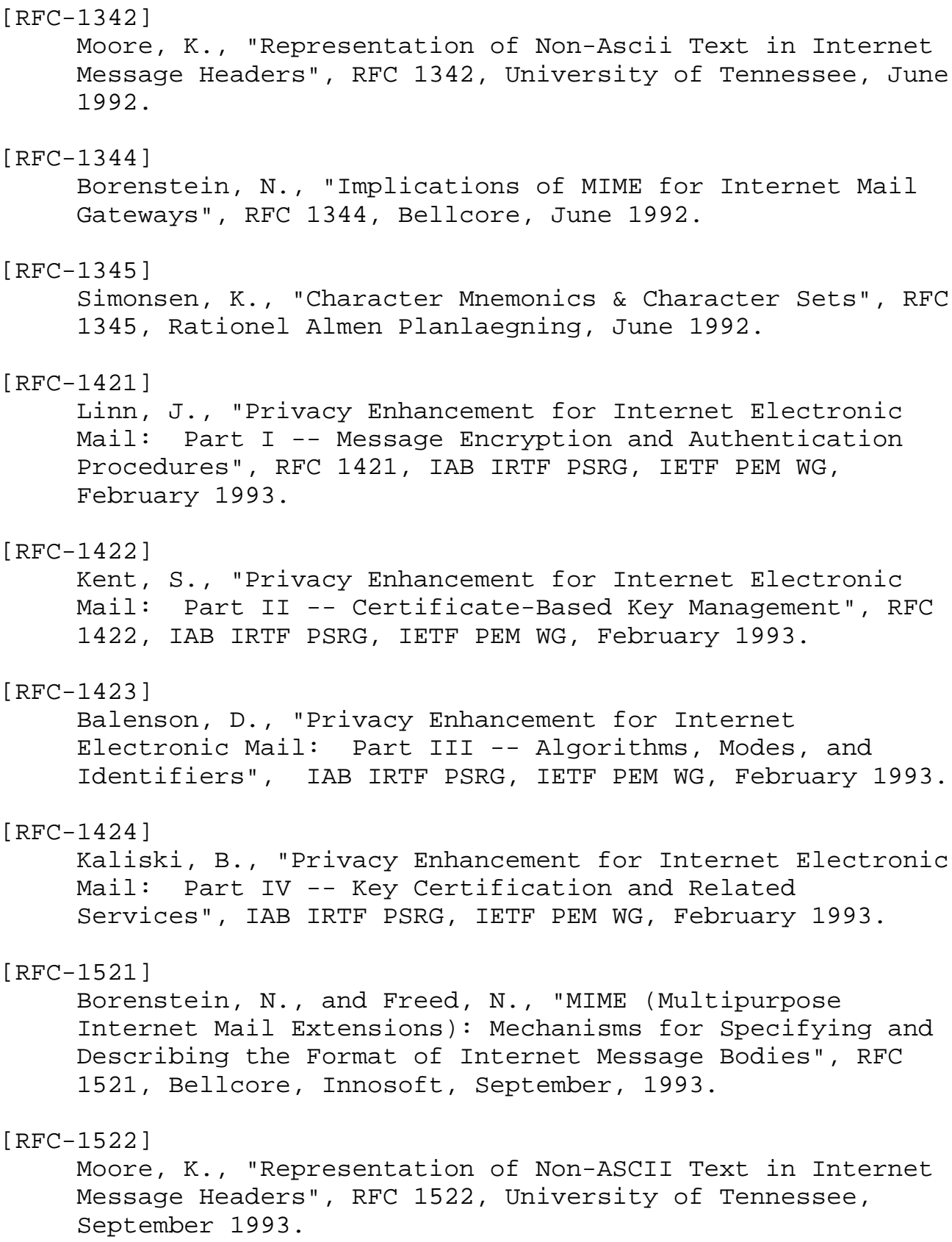




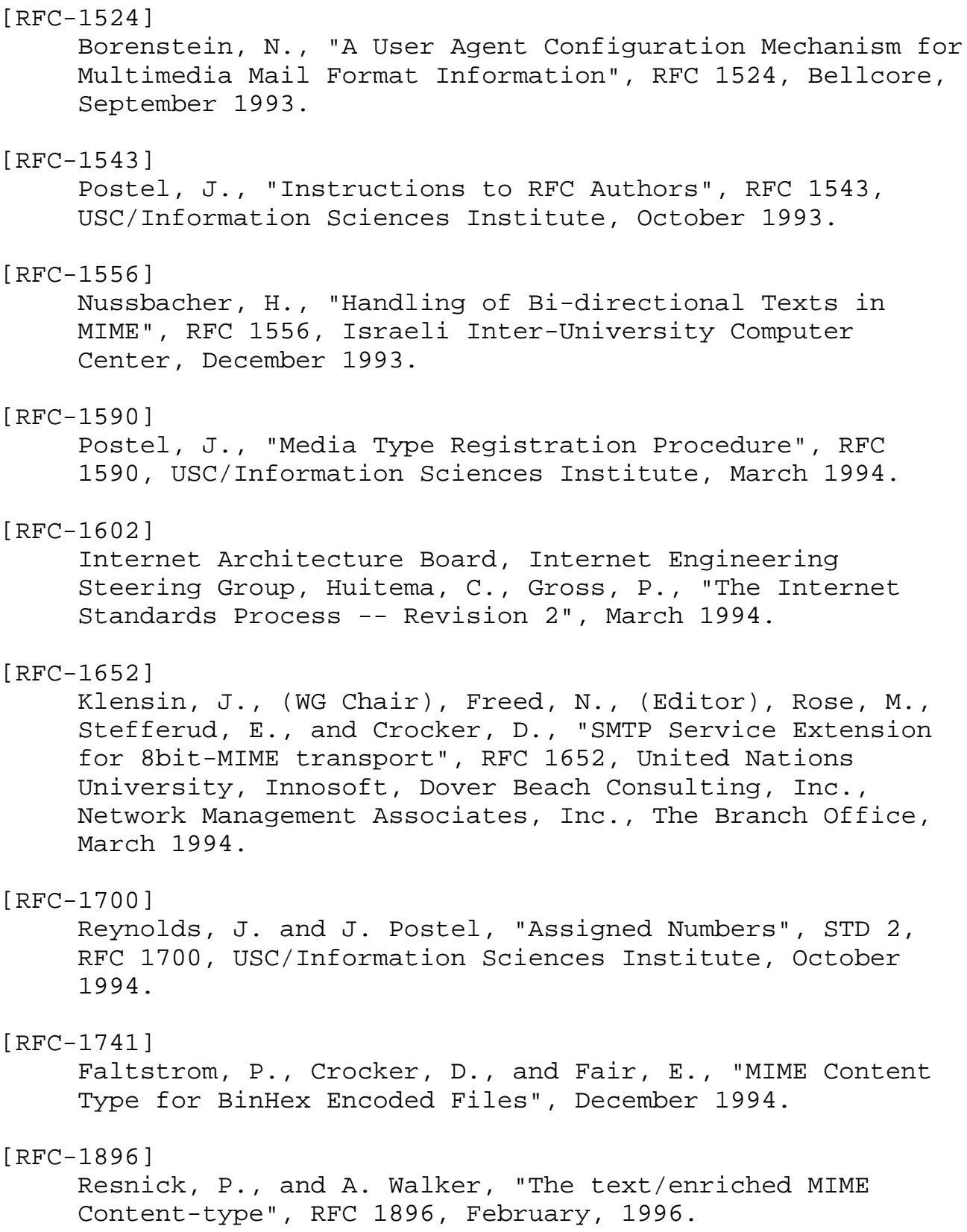




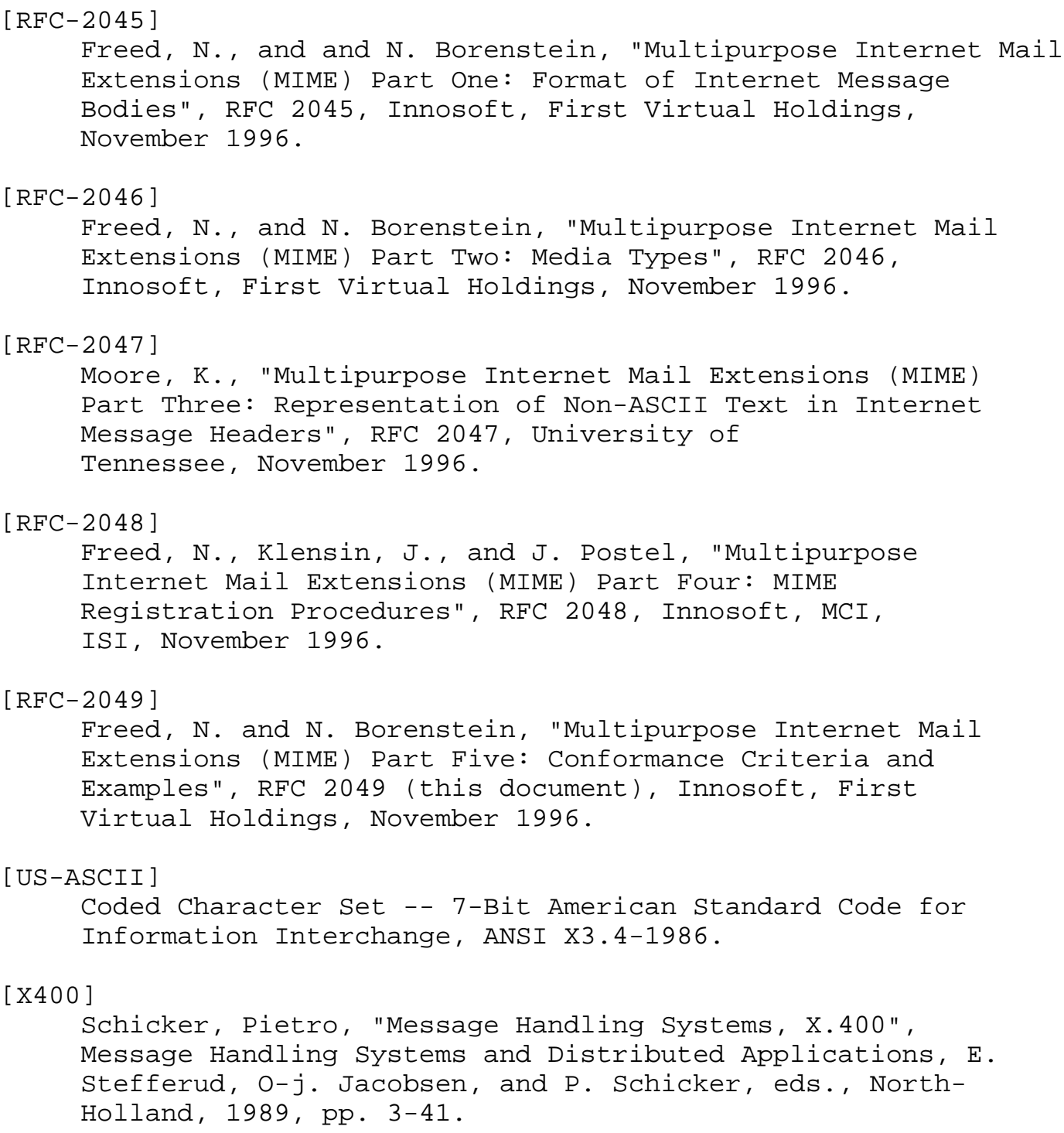

